\title{
A shorter proof of Kanter's Bessel function concentration bound
}

\author{
Lutz Mattner \& Bero Roos \\ Universität zu Lübeck \& Universität Hamburg
}

23rd October 2006

\begin{abstract}
We give a shorter proof of Kanter's (1976) sharp Bessel function bound for concentrations of sums of independent symmetric random vectors. We provide sharp upper bounds for the sum of modified Bessel functions $\mathrm{I}_{0}(x)+\mathrm{I}_{1}(x)$, which might be of independent interest. Corollaries improve concentration or smoothness bounds for sums of independent random variables due to Čekanavičius \& Roos (2006), Roos (2005), Barbour \& Xia (1999), and Le Cam (1986).
\end{abstract}

\section{Introduction}

The principal purpose of this paper is to shorten one proof in the development of Kanter's (1976) remarkable concentration bounds for sums of independent random vectors. Restricting our attention to the symmetric and finite-dimensional case, Kanter's main results may be stated as follows.

Let us call a random vector $X$ symmetrically distributed if $X$ and $-X$ have the same distribution. Let $\|\cdot\|$ be a seminorm on a finite-dimensional $\mathbb{R}$-vector space $E$, let $X_{1}, \ldots, X_{n}$ be independent and symmetrically distributed $E$-valued random vectors, let $t \in] 0, \infty[$, and let us put

$$
p_{j}:=\mathbb{P}\left(\left\|X_{j}\right\| \geq t\right) \quad(j=1, \ldots, n)
$$

Let $Y_{1}, \ldots, Y_{n}$ be independent and symmetrically distributed $\mathbb{R}$-valued random variables with

$$
\mathbb{P}\left(\left|Y_{j}\right|=1\right)=1-\mathbb{P}\left(Y_{j}=0\right)=p_{j} \quad(j=1, \ldots, n)
$$

Let further

$$
G(\lambda):=\mathrm{e}^{-\lambda}\left(\mathrm{I}_{0}(\lambda)+\mathrm{I}_{1}(\lambda)\right) \quad(\lambda \in[0, \infty[)
$$

where $\mathrm{I}_{k}$ denotes the modified Bessel function of order $k$. Then the following three results hold.

\footnotetext{
${ }^{0}$ 2000 Mathematics Subject Classification: 60E15, 60G50, 33C10, $26 \mathrm{D} 07$.

${ }^{0}$ Keywords and phrases: analytic inequalities, Bernoulli convolution, modified Bessel function, concentration function, Poisson binomial distribution, symmetric three point convolution, symmetrized Poisson distribution.
} 


\subsection{Theorem (Kanter's Lemma 4.2).}

$$
\mathbb{P}\left(\left\|\sum_{j=1}^{n} X_{j}-x\right\|<t\right) \leq \mathbb{P}\left(\left|\sum_{j=1}^{n} Y_{j}-\frac{1}{2}\right|<1\right) \quad(x \in E)
$$

1.2 Theorem (Kanter's Lemma 4.3). If $p_{j}>0$ for some $j$, then

$$
\mathbb{P}\left(\left|\sum_{j=1}^{n} Y_{j}-\frac{1}{2}\right|<1\right)<G\left(\sum_{j=1}^{n} p_{j}\right)
$$

1.3 Corollary (Kanter's Theorem 4.1). If $\mathbb{P}\left(\mid X_{j} \|<t\right)<1$ for some $j$, then

$$
\sup _{x \in E} \mathbb{P}\left(\left\|\sum_{j=1}^{n} X_{j}-x\right\|<t\right)<G\left(\sum_{j=1}^{n}\left(1-\mathbb{P}\left(\left\|X_{j}\right\|<t\right)\right)\right)
$$

All three results are optimal: In Theorem 1.1, equality obviously holds whenever $x_{0} \in$ $E$ has norm $t, x=x_{0} / 2$, and $\mathbb{P}\left(X_{j}=x_{0}\right)=\mathbb{P}\left(X_{j}=-x_{0}\right)=p_{j} / 2=\left(1-\mathbb{P}\left(X_{j}=0\right)\right) / 2$ for each $j$. Thus Theorem 1.1 provides the supremum of the left hand side of (4) given the probabilities $p_{j}$ from (1). See Mattner (2006) for a generalization. In Theorem 1.2, the left hand side of (15), being some rather complicated function of $\left(p_{1}, \ldots, p_{n}\right) \in \bigcup_{n \in \mathbb{N}}[0,1]^{n}$, is bounded by its supremum given $\sum_{1}^{n} p_{j}$, see Remark 2.2 (a) below. It follows that Corollary 1.3 provides the supremum of the left hand side in (6) given $\sum_{j=1}^{n}\left(1-\mathbb{P}\left(\left\|X_{j}\right\|<t\right)\right)$.

To further simplify the bounds from (5) or (6), one may use the inequalities from (8), (9) or (11) of the following collection of analytical properties of the function $G$.

1.4 Lemma. $G$ is completely monotone on $\left[0, \infty\left[\right.\right.$, with $G(0)=1$ and $\lim _{\lambda \rightarrow \infty} G(\lambda)=$ 0 . For $\lambda \in] 0, \infty[$, we have

$$
\begin{aligned}
& G(\lambda)=\sqrt{\frac{2}{\pi \lambda}}\left(1-\frac{1}{8 \lambda}-\frac{3}{128 \lambda^{2}}+O\left(\frac{1}{\lambda^{3}}\right)\right) \\
& G(\lambda)<\sqrt{\frac{2}{\pi \lambda}} \\
& G(\lambda)<\sqrt{\frac{\frac{2}{\pi}}{\frac{1}{4}+\lambda}}=\sqrt{\frac{2}{\pi \lambda}}\left(1-\frac{1}{8 \lambda}+\frac{3}{128 \lambda^{2}}+O\left(\frac{1}{\lambda^{3}}\right)\right) \\
& G(\lambda)=1-\frac{\lambda}{2}+\frac{\lambda^{2}}{4}+O\left(\lambda^{3}\right) \\
& G(\lambda)<\frac{1}{\sqrt{1+\lambda}}=1-\frac{\lambda}{2}+\frac{3 \lambda^{2}}{8}+O\left(\lambda^{3}\right)
\end{aligned}
$$

The present paper is mainly concerned with Theorem 1.2. We found Kanter's original proof rather long and somewhat difficult to read, while a much shorter proof of a stronger claim proposed by Marshall \& Olkin (1979) is unfortunately not generally valid, see Remark 2.2|(f) below. Our proof of Theorem 1.2 given below is shorter than Kanter's, and we hope that it is reasonably easy to read. As Theorem 1.2 can be read as an inequality between certain special convolution products and their limits, we have chosen to give an introduction from that point of view in Section 2 below, where Theorem 1.2 
reappears as the slightly more general Theorem 2.1. To check that the latter implies the former, use (201) with $k=0$, (15), and the fact that $\sum_{j=1}^{n} Y_{j}$ from (15) has the distribution $\mathrm{Q}_{p}$ introduced at the beginning of Section 2. This latter fact together with (24) also shows that Theorem 1.1 is indeed a reformulation of Kanter's Lemma 4.2.

Theorem 2.1 is proved in 3.2 below, relying on a crucial analytic inequality provided in 3.1. The rest of Section 3 is then devoted to proofs of Lemma 1.4 and Corollaries 1.5 and [1.6 stated below, which are not needed for the proof of Theorem 2.1.

It is not the purpose of this paper to treat systematically concentration function bounds related to Kanter's. See Petrov (1995) for a good introduction to the onedimensional case. A complete review would include in particular Bretagnolle (2004) and many references from the journal Theory of Probability and Its Applications, such as Rogozin (1993). Let us however give the following corollary to Theorem 1.2 and Lemma 1.4 on concentration functions $\operatorname{Conc}(X, \cdot)$ of real-valued random variables $X$, defined by

$$
\operatorname{Conc}(X, t):=\sup _{x \in \mathbb{R}} \mathbb{P}(X \in[x, x+t]) \quad(t \in[0, \infty[)
$$

We note that (13) below complements Čekanavičius \& Roos (2006, Lemma 3.6), while (14) simplifies and uniformly improves Roos (2005, Proposition 5) and hence as well Le Cam (1986, p. 411, Theorem 2), and complements the results of Bretagnolle. Let $\mathbf{1}_{A}(x)$ be 1 or 0 according to whether $x \in A$ or $x \notin A$. Random variables $X_{j}$ are no longer supposed to be symmetric and numbers $p_{j}$ may differ from those defined in (11).

1.5 Corollary. Let $X_{1}, \ldots, X_{n}$ be independent $\mathbb{R}$-valued random variables and let $t \in\left[0, \infty\left[\right.\right.$. For $j=1, \ldots, n$, let $h_{j}(y):=\inf \left\{x \in \mathbb{R}: \mathbb{P}\left(X_{j} \leq x\right) \geq y\right\}$ for $\left.y \in\right] 0,1[$ and $p_{j}:=2 \int_{0}^{1 / 2} \mathbf{1}_{] t, \infty[}\left(h_{j}(1-y)-h_{j}(y)\right) \mathrm{d} y$. Then $p_{j} \geq 1-\operatorname{Conc}\left(X_{j}, t\right)$ for every $j$ and

$$
\operatorname{Conc}\left(\sum_{j=1}^{n} X_{j}, t\right) \leq G\left(\sum_{j=1}^{n} p_{j}\right) \leq G\left(\sum_{j=1}^{n}\left(1-\operatorname{Conc}\left(X_{j}, t\right)\right)\right)
$$

and hence in particular

$$
\begin{aligned}
& \operatorname{Conc}\left(\sum_{j=1}^{n} X_{j}, t\right) \leq \sqrt{\frac{2}{\pi}}\left(\frac{1}{4}+\sum_{j=1}^{n} p_{j}\right)^{-1 / 2} \\
& \operatorname{Conc}\left(\sum_{j=1}^{n} X_{j}, t\right) \leq\left(1+\sum_{j=1}^{n}\left(1-\operatorname{Conc}\left(X_{j}, t\right)\right)\right)^{-1 / 2}
\end{aligned}
$$

In (13), the constant $\sqrt{\frac{2}{\pi}}$ is optimal, since for $\mathbb{P}\left(X_{j}=-1\right)=\mathbb{P}\left(X_{j}=1\right)=1 / 2$, we have $p_{j}=1$ and $\operatorname{Conc}\left(\sum_{j=1}^{n} X_{j}, 1\right) \sim \sqrt{2 / \pi}\left(\sum_{j=1}^{n} p_{j}\right)^{-1 / 2}$ for $n \rightarrow \infty$.

Theorem 1.2 and Lemma 1.4 further yield an improvement of a smoothness bound of Barbour \& Xia (1999, Proposition 4.6), who applied it in the context of compound Poisson approximation. We denote the total variation distance between distributions of $\mathbb{Z}$-valued random variables $Z_{1}$ and $Z_{2}$ by $d_{\mathrm{TV}}\left(Z_{1}, Z_{2}\right):=\sup _{A \subseteq \mathbb{Z}}\left|\mathbb{P}\left(Z_{1} \in A\right)-\mathbb{P}\left(Z_{2} \in A\right)\right|$.

1.6 Corollary. Let $X_{1}, \ldots, X_{n}$ be independent $\mathbb{Z}$-valued random variables. Then

$$
d_{\mathrm{TV}}\left(\sum_{j=1}^{n} X_{j}, 1+\sum_{j=1}^{n} X_{j}\right) \leq \sqrt{\frac{2}{\pi}}\left(\frac{1}{4}+\sum_{j=1}^{n}\left(1-d_{\mathrm{TV}}\left(X_{j}, 1+X_{j}\right)\right)\right)^{-1 / 2}
$$

This improves Barbour \& Xia's bound $\left(\sum_{j=1}^{n}\left(1-\max \left(\frac{1}{2}, d_{\mathrm{TV}}\left(X_{j}, 1+X_{j}\right)\right)\right)\right)^{-1 / 2}$. 


\section{Symmetric three point convolutions and related distributions}

We let $\mathbb{N}:=\{1,2,3, \ldots\}$ and $\mathbb{N}_{0}:=\{0\} \cup \mathbb{N}$. Throughout the paper, we assume $n \in \mathbb{N}_{0}$ and $p=\left(p_{1}, \ldots, p_{n}\right) \in \mathcal{P}:=\bigcup_{n \in \mathbb{N}_{0}}[0,1]^{n}$ and put $|p|:=\sum_{j=1}^{n} p_{j}, \bar{p}:=|p| / n$, $p_{\text {max }}:=\max _{j=1}^{n} p_{j}$, and $n(p):=n$. For $\alpha \in[0,1], p \in \mathcal{P}$ and $\lambda \in[0, \infty[$, we consider the following probability distributions on $\mathbb{Z}$ :

Bernoulli:

$$
\mathrm{B}_{\alpha}:=(1-\alpha) \delta_{0}+\alpha \delta_{1}
$$

Bernoulli convolution:

$$
\mathrm{B}_{p}:=\underset{j=1}{*} \mathrm{~B}_{p_{j}}
$$

Binomial:

$$
\mathrm{B}_{n, \alpha}:=\mathrm{B}_{\alpha}^{* n}=\mathrm{B}_{(\alpha, \ldots, \alpha)}
$$

Poisson:

$$
\mathrm{P}_{\lambda}:=\sum_{j=0}^{\infty} \mathrm{e}^{-\lambda} \frac{\lambda^{j}}{j !} \delta_{j}
$$

Symmetric 3-point:

$$
\mathrm{Q}_{\alpha}:=(1-\alpha) \delta_{0}+\frac{\alpha}{2}\left(\delta_{-1}+\delta_{1}\right)
$$

Symmetric 3-pt. convolution:

$$
\mathrm{Q}_{p}:=\underset{j=1}{*} \mathrm{Q}_{p_{j}}
$$

Rademacher convolution:

$$
\mathrm{R}_{n}:=\left(\frac{\delta_{-1}+\delta_{1}}{2}\right)^{* n}=\mathrm{Q}_{(1, \ldots, 1)}=\sum_{j=0}^{n}\left(\begin{array}{l}
n \\
j
\end{array}\right) 2^{-n} \delta_{2 j-n}
$$

Symmetrized Poisson:

$$
\mathrm{S}_{\lambda}:=\mathrm{P}_{\lambda / 2} * \widetilde{\mathrm{P}}_{\lambda / 2}=\sum_{j \in \mathbb{Z}} \mathrm{e}^{-\lambda} \mathrm{I}_{j}(\lambda) \delta_{j}
$$

We use roman letters for these special probability distributions, reserving the italic letters $P$ and $Q$ for arbitrary distributions on $\mathbb{Z}$. Here and in what follows, $\widetilde{P}$ denotes the reflection of $P$, defined by $\widetilde{P}(\{k\}):=P(\{-k\}), \delta_{k}$ is the Dirac measure concentrated in $k$, * denotes convolution, and empty convolution products and powers are understood to be $\delta_{0}$. The last representation for the symmetrized Poisson distributions $\mathrm{S}_{\lambda}$ follows from the power series expansion of the modified Bessel functions $\mathrm{I}_{j}$, namely $\mathrm{I}_{j}(x)=\mathrm{I}_{|j|}(x)=$ $\sum_{k=0}^{\infty} \frac{1}{k !(k+|j|) !}\left(\frac{x}{2}\right)^{2 k+|j|}$ for $j \in \mathbb{Z}$ and $x \in \mathbb{C}$, see for example Olver (1997, p. 60). Recalling (3), we observe that

$$
G(\lambda)=\mathrm{S}_{\lambda}(\{0,1\}) \quad(\lambda \in[0, \infty[)
$$

The objects of our study are the symmetric 3-point convolutions $\mathrm{Q}_{p}$. Let us collect some simple properties of these. For every fixed $n$, the probability measure $\mathrm{Q}_{p}$ is a permutation invariant and multiaffine function of the parameter $p \in[0,1]^{n}$. Hence, as noted by Hoeffding (1956, p. 713), each $\mathrm{Q}_{p}$ admits a representation as an expectation with respect to a unique Bernoulli convolution $\mathrm{B}_{p}$. In fact, if we put $P:=\delta_{0}$ and $Q:=$ $\frac{1}{2}\left(\delta_{-1}+\delta_{1}\right)$ in the identity

$$
\underset{j=1}{*}\left(\left(1-p_{j}\right) P+p_{j} Q\right)=\sum_{m=0}^{n} \mathrm{~B}_{p}(\{m\}) Q^{* m} * P^{*(n-m)}
$$

we get

$$
\mathrm{Q}_{p}=\int_{\mathbb{N}_{0}} \mathrm{R}_{m} \mathrm{~dB}_{p}(m) \quad(p \in \mathcal{P})
$$


as a mixture of Rademacher convolutions. In case of $p_{\max } \leq 1 / 2$, the symmetric 3 -point convolution $\mathrm{Q}_{p}$ is also a symmetrized Bernoulli convolution, since

$$
\mathrm{Q}_{\alpha}=\mathrm{B}_{\beta(\alpha)} * \widetilde{\mathrm{B}}_{\beta(\alpha)} \quad \text { for } \quad \alpha \in[0,1 / 2] \quad \text { and } \quad \beta(\alpha):=\frac{1-\sqrt{1-2 \alpha}}{2}
$$

and hence

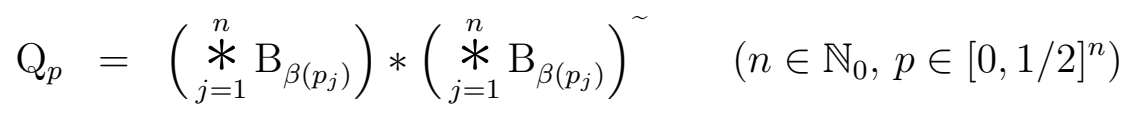

Starting from this representation, one can easily show for every $\lambda \in\left[0, \infty\left[\right.\right.$ that $\mathrm{Q}_{p}$ converges to $\mathrm{S}_{\lambda}$ in total variation if $p \in \mathcal{P}$ varies in such a way that $|p| \rightarrow \lambda$ and $p_{\max } \rightarrow 0$ :

$$
\lim _{|p| \rightarrow \lambda, p_{\max } \rightarrow 0} \mathrm{Q}_{p}=\mathrm{S}_{\lambda} \quad(\lambda \in[0, \infty[)
$$

To verify this, one may use a classical Poisson approximation theorem for Bernoulli convolutions, for example Barbour et al. (1992, p. 3, Le Cam's result (1.6)), the fact that $\beta\left(p_{j}\right)=\left(1+O\left(p_{\max }\right)\right) \frac{p_{j}}{2}$ uniformly in $j$, and the continuity of the map $\lambda \mapsto \mathrm{P}_{\lambda}$. Below we will also need the representation

$$
\mathrm{S}_{\lambda}=\int_{\mathbb{N}_{0}} \mathrm{R}_{m} \mathrm{dP}_{\lambda}(m) \quad(\lambda \in[0, \infty[)
$$

This can be proved similarly to (16) by a short computation or, alternatively, by combining (16) with (18).

Theorem 1.2 is related to, but decidedly less obvious than (18). Rephrased with the notation of this section, it states that $\mathrm{S}_{\lambda}$ with $\lambda=|p|$ even serves as a bound for, and not merely as an approximation to $\mathrm{Q}_{p}$, as far as the concentration over two-point intervals in $\mathbb{Z}$ is concerned.

2.1 Theorem (= Theorem 1.2). Let $p \in \mathcal{P}$ with $|p|>0$ and let $k \in \mathbb{Z}$. Then

$$
\mathrm{Q}_{p}(\{k, k+1\})<\mathrm{S}_{|p|}(\{0,1\})
$$

\subsection{Remarks.}

(a) Inequality (20) is sharp: For every fixed $\lambda>0$, the supremum of the left hand side of (20) over all $p \in \mathcal{P}$ with $|p|=\lambda$ and all $k$ is $\mathrm{S}_{\lambda}(\{0,1\})$, as follows using (18).

(b) As a function of $k \in \mathbb{Z}$, each of $\mathrm{Q}_{p}(\{k, k+1\})$ and $\mathrm{S}_{\lambda}(\{k, k+1\})$ becomes maximal iff $k=0$ or $k=-1$. This follows from the mixture representations (16) and (19), since $\mathrm{R}_{m}(\{k, k+1\})$ obviously becomes maximal iff $k=0$ or $k=-1$. Hence we may rewrite Theorem 2.1 as the concentration function inequality

$$
\sup _{k \in \mathbb{Z}} \mathrm{Q}_{p}(\{k, k+1\})<\sup _{k \in \mathbb{Z}} \mathrm{S}_{|p|}(\{k, k+1\}) \quad(p \in \mathcal{P},|p|>0)
$$

(c) The distributions $S_{\lambda}$ are symmetric and, if $\lambda>0$, strictly unimodal on $\mathbb{Z}$, that is, we have $\mathrm{S}_{\lambda}(\{k\})=\mathrm{S}_{\lambda}(\{|k|\})>\mathrm{S}_{\lambda}(\{|k|+1\})$ for every $k \in \mathbb{Z}$. Here the claimed inequality is a special case of the known strict antitonicity in the order $\nu \in] 0, \infty[$ of the modified Bessel functions $\mathrm{I}_{\nu}(x)$ at fixed arguments $\left.x \in\right] 0, \infty[$, see Olver (1997, p. 251, Theorem 8.1(ii)). For $p \in[0,2 / 3]^{n}$, each $\mathrm{Q}_{p_{j}}$ is symmetric and unimodal on $\mathbb{Z}$, and hence so is their convolution $\mathrm{Q}_{p}$, by the discrete Wintner theorem in Dharmadhikari \& Joag-Dev (1988, p. 109, Theorem 4.7). 
(d) Inequality (21) for two-point intervals in $\mathbb{Z}$ does not generalize in the obvious way to more general $\ell$-point intervals $\{k, \ldots, k+\ell-1\}$ with $\ell \in \mathbb{N}$. More precisely, there is no $\ell \in \mathbb{N} \backslash\{2\}$ such that the inequality

$$
\sup _{k \in \mathbb{Z}} \mathrm{Q}_{p}(\{k, \ldots, k+\ell-1\}) \leq \sup _{k \in \mathbb{Z}} \mathrm{S}_{|p|}(\{k, \ldots, k+\ell-1\})
$$

holds for every $p \in \mathcal{P}$. For $\ell=1$, we get a counterexample to (22) by choosing $p=(1, \ldots, 1) \in\{1\}^{n}$ with $n:=2 m$, for large enough $m \in \mathbb{N}$. In fact, for $m \rightarrow \infty$, we have $\mathrm{Q}_{p}(\{0\})=\mathrm{R}_{2 m}(\{0\})=\left(\begin{array}{c}2 m \\ m\end{array}\right) 2^{-2 m} \sim 2 / \sqrt{2 \pi n}$ but, using Remark (c), we have $\sup _{k \in \mathbb{Z}} \mathrm{S}_{n}(\{k\})=\mathrm{S}_{n}(\{0\})=\mathrm{e}^{-n} \mathrm{I}_{0}(n) \sim 1 / \sqrt{2 \pi n}$. See Olver (1997, p. 83) or (35) below for the standard Bessel function asymptotics just used. For $\ell \geq 3$, we get a trivial counterexample by choosing $p \in] 0,1]$ one-dimensional, since then the left hand side of (22) equals 1. More generally,

$$
G_{\ell}(\lambda):=\sup \left\{\sup _{k \in \mathbb{Z}} Q_{p}(\{k, \ldots, k+\ell-1\}): p \in \mathcal{P},|p|=\lambda\right\} \quad(\ell \in \mathbb{N}, \lambda \in[0, \infty[)
$$

equals 1 for $\lambda \leq\lfloor(\ell-1) / 2\rfloor$, where, as usual, $\lfloor x\rfloor:=\sup \{n \in \mathbb{Z}: n \leq x\}$. While Theorem 2.1 and Remark (a) state that $G_{2}$ is just $G$ from (3) and (15), it is an open problem to compute $G_{\ell}(\lambda)$ for $\ell \in \mathbb{N} \backslash\{2\}$ and $\lambda>\lfloor(\ell-1) / 2\rfloor$.

(e) Applying (16) and (19) to the event $\{0,1\}$, and writing

$$
\psi(m):=\mathrm{R}_{m}(\{0,1\})=\left(\begin{array}{c}
m \\
\left\lfloor\frac{m+1}{2}\right\rfloor
\end{array}\right) 2^{-m}=\left(\begin{array}{c}
2\left\lfloor\frac{m+1}{2}\right\rfloor \\
\left\lfloor\frac{m+1}{2}\right\rfloor
\end{array}\right) 2^{-2\left\lfloor\frac{m+1}{2}\right\rfloor} \quad\left(m \in \mathbb{N}_{0}\right)
$$

we get, using the notation $P \varphi:=\int \varphi \mathrm{d} P$,

$$
\begin{array}{ll}
\mathrm{Q}_{p}(\{0,1\})=\mathrm{B}_{p} \psi & (p \in \mathcal{P}) \\
\mathrm{S}_{\lambda}(\{0,1\})=\mathrm{P}_{\lambda} \psi & (\lambda \in[0, \infty[)
\end{array}
$$

so that Theorem 2.1 can also be stated as

$$
\mathrm{B}_{p} \psi<\mathrm{P}_{|p|} \psi \quad(p \in \mathcal{P},|p|>0)
$$

Using most conveniently the last expression for $\psi$ from (23), we compute

$$
\psi(0)=1, \quad \psi(1)=\psi(2)=\frac{1}{2}, \quad \psi(3)=\psi(4)=\frac{3}{8}
$$

(f) Marshall \& Olkin (1979, p. 378, Theorem K.4, the case $m=1$ ) claim that $\mathrm{Q}_{p}(\{0,1\})$ is, for fixed $n \in \mathbb{N}$, a Schur-concave function of $p \in[0,1]^{n}$. Their proof and claim become correct if " $p \in[0,1]^{n "}$ is replaced by " $p \in[0,1 / 2]^{n}$ ". Without this change their claim is false. To see this, let us note that the following three properties of functions $\varphi: \mathbb{N}_{0} \rightarrow \mathbb{R}$ are equivalent:

(i) $\varphi$ is convex, that is, $\varphi(k+2)-2 \varphi(k+1)+\varphi(k) \geq 0$ holds for $k \in \mathbb{N}_{0}$,

(ii) for every $n \in \mathbb{N}$, the Bernoulli convolution expectation $\mathrm{B}_{p} \varphi$ is a Schur concave function of $p \in[0,1]^{n}$,

(iii) $\mathrm{B}_{p} \varphi \leq \mathrm{B}_{n, \bar{p}} \varphi$ for every $n \in \mathbb{N}$ and $p \in[0,1]^{n}$.

Here the implication "(i) $\Rightarrow$ (ii)" is due to Karlin \& Novikoff (1963, pp. 1257-1258), "(ii) $\Rightarrow$ (iii)" is clear, and "(iii) $\Rightarrow$ (i)" can be seen as follows: For given $k \in \mathbb{N}$, let $n:=k+$ $2, \varepsilon \in] 0,1 / 2]$, and $p:=(1-\varepsilon, \ldots, 1-\varepsilon, 1,1-2 \varepsilon) \in[0,1]^{n}$, so that $\mathrm{B}_{p}=\mathrm{B}_{k, 1-\varepsilon} * \delta_{1} * \mathrm{~B}_{1-2 \varepsilon}$. 
Then $\bar{p}=1-\varepsilon$ and $\mathrm{B}_{n, \bar{p}}-\mathrm{B}_{p}=\varepsilon^{2} \mathrm{~B}_{k, 1-\varepsilon} *\left(\delta_{0}-2 \delta_{1}+\delta_{2}\right)=\varepsilon^{2}\left(\delta_{k}-2 \delta_{k+1}+\delta_{k+2}\right)+O\left(\varepsilon^{3}\right)$, so that $\mathrm{B}_{p} \varphi \leq \mathrm{B}_{n, \bar{p}} \varphi$ for every $\varepsilon$ implies $\varphi(k+2)-2 \varphi(k+1)+\varphi(k) \geq 0$. We remark that, similarly, (ii) with $n$ fixed implies the convexity of $\left.\varphi\right|_{\{0, \ldots, n\}}$.

We refer to Hoeffding (1956, Theorem 3) for the original proof of "(i) $\Rightarrow$ (iii)", to Gleser (1975) and Boland \& Proschan (1983) for generalizations of "(i) $\Rightarrow$ (ii)" under restrictions on $p$, and to Bickel \& van Zwet (1980) and Berg et al. (1984, Chapter 7) for results involving distributions more general than Bernoulli convolutions.

Coming back to the claim of Marshall \& Olkin, we observe that the function $\psi$ from (23) and (27) is not convex on $\{0,1,2,3\}$, so that it follows, via (24) and using the remark after the above proof of "(iii) $\Rightarrow$ (i)", that $\mathrm{Q}_{p}(\{0,1\})$ is not a Schur concave function of $p \in[0,1]^{n}$ in case $n \geq 3$.

Let us finally note that Bondar (1994) gives valuable comments on and corrections to Marshall \& Olkin (1979) in general, but does not refer to their claim cited above, while Merkle \& Petrović (1997, p. 175) repeat the claim and attribute it to Kanter (1976).

(g) The Poisson bound $\mathrm{P}_{|p|} \psi$ on the right in (26) can not in general be replaced by the binomial bound $\mathrm{B}_{n(p), \bar{p}} \psi$. This follows from the implication "(iii) $\Rightarrow$ (i)" of the preceding remark.

\section{Proofs}

3.1 A special analytic inequality. Let $\lambda \in] 0, \infty[, \alpha \in] 0,1]$, and

$$
F(\lambda, \alpha):=\frac{1}{\pi} \int_{0}^{\pi}|1-\alpha(1-\cos t)|^{\frac{\lambda}{\alpha}}(1+\cos t) \mathrm{d} t
$$

Then

$$
F(\lambda, \alpha)<G(\lambda)
$$

Remark. It easily follows that $\sup _{\alpha \in] 0,1]} F(\lambda, \alpha)=\lim _{\alpha \downarrow 0} F(\lambda, \alpha)=G(\lambda)$, using (30).

Proof. By Olver (1997, p. 60) or by (15) and Fourier inversion of $\mathrm{S}_{\lambda}$, we have

$$
G(\lambda)=\frac{1}{\pi} \int_{0}^{\pi} \mathrm{e}^{-\lambda(1-\cos t)}(1+\cos t) \mathrm{d} t
$$

If $\alpha \in] 0,1 / 2]$, then $|1-\alpha(1-\cos t)|=1-\alpha(1-\cos t)<\exp (-\alpha(1-\cos t))$ for every $t \in] 0, \pi]$, yielding (29).

So let $\left.\alpha \in] \frac{1}{2}, 1\right]$. We split the integral from (28) as $\int_{0}^{t(\alpha)}+\int_{t(\alpha)}^{\pi}$ with $t(\alpha):=\arccos (1-$ $\left.\frac{1}{\alpha}\right)$, substitute $t=\arccos \left(1-\frac{1}{\alpha}\left(1-\mathrm{e}^{-x}\right)\right)$ in the first integral, and $t=\arccos \left(1-\frac{1}{\alpha}\left(1+\mathrm{e}^{-x}\right)\right)$ in the second. We also rewrite $G$ by substituting $t=\arccos (1-x / \alpha)$ in the integral from (30). Thus, with the abbreviations

$$
\begin{aligned}
\beta & :=2 \alpha-1 \in] 0,1] \\
x_{\alpha} & :=-\log \beta \in[0, \infty[ \\
f(x) & :=\frac{\mathrm{e}^{-x}}{\pi \alpha} \sqrt{\frac{\beta+\mathrm{e}^{-x}}{1-\mathrm{e}^{-x}}}+\frac{\mathrm{e}^{-x}}{\pi \alpha} \sqrt{\frac{\beta-\mathrm{e}^{-x}}{1+\mathrm{e}^{-x}}} \mathbf{1}_{] x_{\alpha}, \infty[}(x) \\
g(x) & :=\frac{1}{\pi \alpha} \sqrt{\frac{2 \alpha-x}{x}} \mathbf{1}_{] 0,2 \alpha[}(x)
\end{aligned}
$$


for $x \in] 0, \infty[$, we get

$$
F(\lambda, \alpha)=\int_{0}^{\infty} \exp \left(-\frac{\lambda x}{\alpha}\right) f(x) \mathrm{d} x, \quad G(\lambda)=\int_{0}^{\infty} \exp \left(-\frac{\lambda x}{\alpha}\right) g(x) \mathrm{d} x
$$

By letting $\lambda$ tend to 0 in (28), (30) and (31), we conclude that $\int_{0}^{\infty} f \mathrm{~d} x=\int_{0}^{\infty} g \mathrm{~d} x=1$. Let us assume the following claim for the moment.

Claim. There is a $\left.y_{\alpha} \in\right] 0, \infty[$ with $f<g$ on $] 0, y_{\alpha}[$ and $f>g$ on $] y_{\alpha}, \infty[$.

Then, with $\varphi(x):=\exp (-\lambda x / \alpha)$ being strictly decreasing in $x \in] 0, \infty[$, we get $\int_{0}^{\infty} \varphi f \mathrm{~d} x<\int_{0}^{\infty} \varphi g \mathrm{~d} x$, and hence (29), by integrating the inequality $\left(\varphi(x)-\varphi\left(y_{\alpha}\right)\right)(f(x)-$ $g(x))<0$ over $x \in] 0, \infty\left[\backslash\left\{y_{\alpha}\right\}\right.$.

Thus it only remains to prove the claim, which will be reduced to two subclaims. Let us agree to use interval notation like $] a, b]:=\{x \in \mathbb{R}: a<x \leq b\}$ also if $a=b$ and even if $a>b$.

SUBCLAIm 1. If $x_{\alpha}>0$, then there is a $\left.\left.u_{\alpha} \in\right] 0, x_{\alpha}\right]$ with $f<g$ on $] 0, u_{\alpha}[$ and $f>g$ on $\left.] u_{\alpha}, x_{\alpha}\right]$.

SubClaim 2. There is a $v_{\alpha} \in\left[x_{\alpha}, \infty\left[\right.\right.$ with $f<g$ on $\left[x_{\alpha}, v_{\alpha}\lfloor\backslash\{0\}\right.$ and $f>g$ on ]$v_{\alpha}, \infty[$.

Proof of the Claim ASsuming the two subClaims. If $x_{\alpha}=0$, then we put $y_{\alpha}:=v_{\alpha}$ and observe that here $v_{\alpha}>0$, since both $f$ and $g$ integrate to 1 over $] 0, \infty[$. So let us assume now that $x_{\alpha}>0$. For $u_{\alpha}<x_{\alpha}$, Subclaim 1 yields $f\left(x_{\alpha}\right)>g\left(x_{\alpha}\right)$, while for $v_{\alpha}>x_{\alpha}$, Subclaim 2 yields $f\left(x_{\alpha}\right)<g\left(x_{\alpha}\right)$. Hence $u_{\alpha}=x_{\alpha}$ or $v_{\alpha}=x_{\alpha}$. We put $y_{\alpha}:=v_{\alpha}$ in the first case and $y_{\alpha}:=u_{\alpha}$ in the second. This proves the claim.

Proof of Subclaim 1. For $\left.x \in] \min \left(x_{\alpha}, 2 \alpha\right), x_{\alpha}\right]$, we have $f(x)-g(x)=f(x)>0$, while for $x \in] 0, \min \left(x_{\alpha}, 2 \alpha\right)$ ], the difference $f(x)-g(x)$ has the same sign as

$$
\eta(x):=\frac{\left(f^{2}(x)-g^{2}(x)\right)(\pi \alpha)^{2} x \mathrm{e}^{2 x}\left(1-\mathrm{e}^{-x}\right)}{\mathrm{e}^{2 x}-\mathrm{e}^{x}-x}=\frac{x\left(\mathrm{e}^{2 x}-\mathrm{e}^{x}+\mathrm{e}^{-x}-1\right)}{\mathrm{e}^{2 x}-\mathrm{e}^{x}-x}-2 \alpha
$$

Differentiating $\eta$ according to the quotient rule yields a fraction with a positive denominator and with numerator

$$
\mathrm{e}^{4 x}-2 \mathrm{e}^{3 x}+\left(-2 x^{2}+2 x\right) \mathrm{e}^{2 x}+\left(x^{2}-4 x+2\right) \mathrm{e}^{x}+2 x-1+x^{2} \mathrm{e}^{-x}=\sum_{k=2}^{\infty} \frac{(4 x)^{k}}{k !} a_{k}>0
$$

where $a_{2}=a_{3}=0$, while for $k \geq 4$

$$
\begin{aligned}
a_{k} & :=1-2\left(\frac{3}{4}\right)^{k}-\frac{k(k-3)}{2^{k+1}}+\frac{k(k-1)\left(1+(-1)^{k}\right)-4 k+2}{4^{k}} \\
& >1-2\left(\frac{3}{4}\right)^{k}-\frac{(k-1)(k-2)}{2^{k+1}}-\frac{k}{4^{k-1}}=: b_{k} \geq b_{4}=\frac{15}{128}>0
\end{aligned}
$$

by the monotonicity of the terms in the definition of $b_{k}$. Thus $\eta$ is strictly increasing, and since $\lim _{x \rightarrow 0} \eta(x)=-2 \alpha<0$, Subclaim 1 follows with $\left.u_{\alpha}:=\sup \{x \in] 0, \min \left(x_{\alpha}, 2 \alpha\right)\right]$ : $\eta(x)<0\}$.

Proof of Subclaim 2. For $x \in\left[x_{\alpha}, \infty[\right.$

$$
f_{1}(x):=\left(\pi \alpha f(x) \sqrt{\mathrm{e}^{2 x}-1}\right)^{2}=2\left(\beta+\mathrm{e}^{-2 x}+\sqrt{\left(\beta^{2}-\mathrm{e}^{-2 x}\right)\left(1-\mathrm{e}^{-2 x}\right)}\right)
$$


is increasing (possibly constant), since for $x \in] x_{\alpha}, \infty[$

$$
f_{1}^{\prime}(x)=-4 \mathrm{e}^{-2 x}\left(1-\frac{\frac{1}{2}\left(1+\beta^{2}\right)-\mathrm{e}^{-2 x}}{\sqrt{\left(\beta^{2}-\mathrm{e}^{-2 x}\right)\left(1-\mathrm{e}^{-2 x}\right)}}\right) \geq 0
$$

by $\sqrt{a b} \leq(a+b) / 2$ with $a=\beta^{2}-\mathrm{e}^{-2 x}$ and $b=1-\mathrm{e}^{-2 x}$. Further, $\lim _{x \rightarrow \infty} f_{1}(x)=4 \beta \leq 4 \alpha$.

For $x \in] 0,2 \alpha[$, let

$$
g_{1}(x):=\left(\pi \alpha g(x) \sqrt{\mathrm{e}^{2 x}-1}\right)^{2}=\frac{\mathrm{e}^{2 x}-1}{x}(2 \alpha-x)=4 \alpha+\sum_{k=1}^{\infty} \frac{(2 x)^{k}}{k !}\left(\frac{4 \alpha}{k+1}-1\right)
$$

Then $g_{1}$ is strictly decreasing on the interval $\{x \in] 0,2 \alpha\left[: g_{1}(x)<4 \alpha\right\}$, since $\lim _{x \downarrow 0} g_{1}(x)=$ $4 \alpha$ and since $g_{1}^{\prime}(x)=: \sum_{k=0}^{\infty} c_{k} x^{k}$ is, for some $\delta>0$, strictly positive on $] 0, \delta[$ and strictly negative for $x>\delta$, as the coefficients $c_{k}=\left(2^{k+1} / k !\right)(4 \alpha /(k+2)-1)$ change sign exactly once, from plus to minus. See Pólya \& Szegö (1971, p. 43, Aufgaben V.38 and V.40) for this last argument.

Hence there is at most one $x$ in the possibly empty interval $\left[x_{\alpha}, 2 \alpha\left[\right.\right.$ with $f_{1}(x)=g_{1}(x)$. We take this $x$, if it exists, as $v_{\alpha}$, and otherwise put $v_{\alpha}:=x_{\alpha}$. The subclaim follows: For $x \in\left[x_{\alpha}, 2 \alpha\left[\backslash\{0\}\right.\right.$, the difference $f(x)-g(x)$ has the same sign as $f_{1}(x)-g_{1}(x)$, while for $x \geq 2 \alpha$, we have $f(x)>0=g(x)$.

3.2 Proof of Theorem 2.1. By Remark 2.2](b), it suffices to prove the claim for $k=0$. Fourier inversion yields

$$
\mathrm{Q}_{p}(\{0,1\})=\frac{1}{\pi} \int_{0}^{\pi}\left(\prod_{j=1}^{n}\left(1-p_{j}(1-\cos t)\right)\right)(1+\cos t) \mathrm{d} t
$$

for $p \in \mathcal{P}$. Let us fix $n \in \mathbb{N}$ and $\lambda \in] 0, n]$ for the rest of this proof. By Hoeffding (1956, Corollary 2.1$)$, the probability $\mathrm{Q}_{p}(\{0,1\})$, being a permutation invariant and multiaffine function of $p \in[0,1]^{n}$, attains its maximum subject to the constraint $|p|=\lambda$ at some point $(1, \ldots, 1, \alpha, \ldots, \alpha, 0, \ldots, 0) \in[0,1]^{n}$ having at most three different coordinate values, with at most one of them distinct from 0 and 1 . Thus, for every $p \in[0,1]^{n}$ with $|p|=\lambda$, there exist $\ell, m \in \mathbb{N}_{0}$ and $\left.\alpha \in\right] 0,1[$ with $\ell+m \leq n, \ell+m \alpha=\lambda$, and

$$
\begin{array}{rlrl}
\mathrm{Q}_{p}(\{0,1\}) & \leq \frac{1}{\pi} \int_{0}^{\pi}(\cos t)^{\ell}(1-\alpha(1-\cos t))^{m}(1+\cos t) \mathrm{d} t & \text { [using (32)] } \\
& \leq \frac{1}{\pi} \int_{0}^{\pi}|\cos t|^{\ell}|1-\alpha(1-\cos t)|^{\frac{\lambda-\ell}{\alpha}}(1+\cos t) \mathrm{d} t \\
& \leq \max (F(\lambda, \alpha), F(\lambda, 1)) & & {[\text { see below and (28)] }]} \\
& <G(\lambda) & & \text { [by Lemma [3.1] } \\
& =\mathrm{S}_{\lambda}(\{0,1\}) & & \text { [by (15) ] }
\end{array}
$$

Here (33) follows by regarding the integrand, and hence the integral, in the preceding line as a convex function of the parameter $\ell \in[0, \lambda]$, so that the integral becomes maximal for $\ell=0$ or $\ell=\lambda$.

3.3 Proof of Lemma 1.4. The Laplace transform representation (31) with $\alpha=1$,

$$
G(\lambda)=\int_{0}^{\infty} \mathrm{e}^{-\lambda x} g(x) \mathrm{d} x, \quad g(x)=\frac{1}{\pi} \sqrt{\frac{2-x}{x}} \mathbf{1}_{] 0,2[}(x)
$$


yields the complete monotonicity of $G$, compare Berg et al. (1984, p. 135).

It is easily checked that there is no need to qualify the big $\mathrm{O}$ claims in the lemma or this proof by " $\lambda \rightarrow \infty$ " or similarly. Standard Bessel function asymptotics, see Olver (1997, pp. 251, 238), yield for every $k \in \mathbb{Z}$

$$
\mathrm{S}_{\lambda}(\{k\})=\mathrm{e}^{-\lambda} \mathrm{I}_{k}(\lambda)=\frac{1}{\sqrt{2 \pi \lambda}}\left(1-\frac{4 k^{2}-1}{8 \lambda}+\frac{\left(4 k^{2}-1\right)\left(4 k^{2}-9\right)}{128 \lambda^{2}}+O\left(\frac{1}{\lambda^{3}}\right)\right)
$$

and hence (77). Also, for fixed $k \in \mathbb{N}_{0}$, a multiplication of power series yields

$$
\mathrm{S}_{\lambda}(\{k\})=\left(\sum_{j=0}^{\infty} \frac{(-\lambda)^{j}}{j !}\right)\left(\sum_{j=0}^{\infty} \frac{(\lambda / 2)^{2 j+k}}{j !(j+k) !}\right)=\frac{\lambda^{k}}{2^{k} k !}-\frac{\lambda^{k+1}}{2^{k} k !}+\frac{(2 k+3) \lambda^{k+2}}{2^{k+2}(k+1) !}+O\left(\lambda^{k+3}\right)
$$

and hence (10). The big $\mathrm{O}$ claims in (9) and (11) are obvious from the binomial series.

For $a, b \in[0, \infty[$, we have

$$
\sqrt{\frac{a}{b+\lambda}}=\int_{0}^{\infty} \mathrm{e}^{-\lambda x} \sqrt{\frac{a}{\pi x}} \mathrm{e}^{-b x} \mathrm{~d} x
$$

Taking here $a=2 / \pi$ and $b=1 / 4$ and using $\mathrm{e}^{-x / 4}=\sqrt{\mathrm{e}^{-x / 2}}>\sqrt{1-\frac{x}{2}} \mathbf{1}_{0,2[}(x)$ and hence $\frac{1}{\pi} \sqrt{\frac{2}{x}} \mathrm{e}^{-x / 4}>g(x)$, compare (34), yields the inequality in (9). Inequality (8) follows trivially. Taking $a=b=1$ in (36) yields

$$
H(\lambda):=\frac{1}{\sqrt{1+\lambda}}=\int_{0}^{\infty} \mathrm{e}^{-\lambda x} h(x) \mathrm{d} x
$$

with $h(x):=\mathrm{e}^{-x} / \sqrt{\pi x}$. Now

$$
\int_{0}^{\infty} h(x) \mathrm{d} x=1=\int_{0}^{\infty} g(x) \mathrm{d} x \text { and } \int_{0}^{\infty} x h(x) \mathrm{d} x=\frac{1}{2}=\int_{0}^{\infty} x g(x) \mathrm{d} x
$$

On ]0,2[, the derivative $(\log (g(x) / h(x)))^{\prime}=\left(\frac{3}{2}-x\right) /(2-x)$ changes sign exactly once, from plus to minus. Further, $\lim _{x \downarrow 0} g(x) / h(x)=\sqrt{2 / \pi}<1, g / h$ is continuous on $] 0, \infty[$, and $g(x)=0<h(x)$ for $x \in[2, \infty[$. Recalling the first half of (37), we deduce the existence of $\left.x_{1}, x_{2} \in\right] 0,2\left[\right.$ with $x_{1}<x_{2}, g<h$ on $] 0, x_{1}[\cup] x_{2}, \infty[$, and $g>h$ on $] x_{1}, x_{2}[$. Thus, since $\varphi(x):=\mathrm{e}^{-\lambda x}$ is strictly convex, we have

$$
\left(\varphi(x)-\left(\varphi\left(x_{1}\right) \frac{x_{2}-x}{x_{2}-x_{1}}+\varphi\left(x_{2}\right) \frac{x-x_{1}}{x_{2}-x_{1}}\right)\right)(h(x)-g(x))>0
$$

for $x \in] 0, \infty\left[\backslash\left\{x_{1}, x_{2}\right\}\right.$, and hence obtain $G(\lambda)<H(\lambda)$ by integration, using (37).

3.4 Proof of Corollary 1.5. Clearly $p_{j} \geq 1-\operatorname{Conc}\left(X_{j}, t\right)$ for every $j$. Hence

$$
\operatorname{Conc}\left(\sum_{j=1}^{n} X_{j}, t\right) \leq \mathrm{B}_{p} \psi=\mathrm{Q}_{p}(\{0,1\}) \leq G(|p|) \leq G\left(\sum_{j=1}^{n}\left(1-\operatorname{Conc}\left(X_{j}, t\right)\right)\right)
$$

by Le Cam (1986, p. 411, Proof of Theorem 2), (24), (20), (15), and the antitonicity of $G$ from Lemma 1.4, Applying (9) and (11) yields the claimed inequalities. 
3.5 Proof of Corollary 1.6. By using the Mineka coupling, Barbour \& Xia (1999, Proposition 4.6) proved that

$$
d_{\mathrm{TV}}\left(\sum_{j=1}^{n} X_{j}, 1+\sum_{j=1}^{n} X_{j}\right) \leq \mathbb{P}\left(\sum_{j=1}^{n} Y_{j} \in\{0,1\}\right)
$$

where the $Y_{1}, \ldots, Y_{n}$ are independent and symmetric as in (2), but now with $p_{j}=1-$ $d_{\mathrm{TV}}\left(X_{j}, 1+X_{j}\right)$ for $j=1, \ldots, n$. Thus Theorem 1.2 and (9) yield our claim.

Acknowledgements. We thank Uwe Rösler for discussions prompting Remark 2.2](f), Bero Roos thanks Professor Wilfried Grecksch of Martin-Luther-University Halle-Wittenberg for an invitation to the most hospitable Institute of Optimization and Stochastics for the Winter term 2005/06, during which this work was finished.

\section{References}

Barbour, A.D., Holst, L. \& Janson, S. (1992). Poisson Approximation. Clarendon Press, Oxford.

Barbour, A.D. \& XiA, A. (1999). Poisson perturbations. ESAIM Probab. Statist. 3, 131-150.

Berg, C., Christensen, J.P.R. \& Ressel, P. (1984). Harmonic Analysis on Semigroups. Springer, New York.

Bickel, P.J. \& van Zwet, W.R. (1980). On a theorem of Hoeffding. In: Asymptotic Theory of Statistical Tests and Estimation: in Honor of Wassily Hoeffding, I.M. Chakravarti (ed.), Academic Press, New York, pages 307-324.

Boland, P.J. \& Proschan, F. (1983). The reliability of $k$ out of $n$ systems. Ann. Probab. 11, 760764 .

Bondar, J.V. (1994). Comments and complements to Inequalities: Theory of Majorization and Its Applications by Albert W. Marshall and Ingram Olkin. Linear Algebra and its Applications 199, $115-130$.

Bretagnolle, J. (2004). Sur l'inégalité de concentration de Doeblin-Lévy, Rogozin-Kesten. In: Parametric and semiparametric models with applications to reliability, survival analysis, and quality of life, 533-551, Stat. Ind. Technol., Birkhäuser, Boston.

Čekanavičius, V. \& Roos, B. (2006). An expansion in the exponent for compound binomial approximations. Lith. Math. J. 46, 54-91.

Dharmadhikari, S. \& Joag-Dev, K. (1988). Unimodality, Convexity, and Applications. Academic Press, Boston.

Gleser, L. (1975). On the distribution of the number of successes in independent trials. Ann. Probab. 3, 182-188.

Hoeffoding, W. (1956). On the distribution of the number of successes in independent trials. Ann. Math. Statist. 27, 713-721. Also in: The Collected Works of Wassily Hoeffding, N.I. Fisher \& P.K. Sen (eds.), Springer, New York, 1994.

KAnter, M. (1976). Probability inequalities for convex sets and multidimensional concentration functions. J. Multivariate Anal. 6, 222-236.

Karlin, S. \& Novikoff, A. (1963). Generalized convex inequalities. Pacific J. Math. 13, 1251-1279.

Le Cam, L. (1986). Asymptotic Methods in Statistical Decision Theory. Springer, New York.

Marshall, A.W. \& Olkin, I. (1979). Inequalities: Theory of Majorization and Its Applications. Academic Press, New York. 
Mattner, L. (2006). Lower bounds for tails of sums of independent symmetric random variables. Preprint, http://arxiv.org/abs/math.PR/0609200.

Merkle, M. \& Petrović, L. (1997). Inequalities for sums independent geometrical random variables. Aequ. Math. 54, 173-180.

Olver, F.W.J. (1997). Asymptotics and Special Functions. A K Peters, Wellesley, Massachusetts.

Petrov, V.V. (1995). Limit Theorems of Probability Theory. Sequences of Independent Random Variables. Clarendon Press, Oxford.

Pólya, G. \& Szegö, G. (1971). Aufgaben und Lehrsätze aus der Analysis II. 4. Auflage, Springer, Berlin.

Rogozin, B.A. (1993). Inequalities for concentration of a decomposition. Theory Probab. Appl. 38, $556-562$.

Roos, B. (2005). On Hipp's compound Poisson approximations via concentration functions. Bernoulli 11, 533-557.

UNIVERSITÄT ZU LÜBECK

INSTITUT FÜR MATHEMATIK

WALLSTR. 40

D-23560 LÜBECK

GERMANY

EMAIL: mattner@math.uni-luebeck.de
UNIVERSITÄT HAMBURG

Department Mathematik, SPST

BundESSTR. 55

D-20146 Hamburg

Germany

EMAIL: roos@math.uni-hamburg.de 